\title{
Second Eye Involvement in Age-Related Macular Degeneration: A Four-Year Prospective Study
}

\author{
MONIQUE ROY and MURIEL KAISER-KUPFER \\ Bethesda, Maryland
}

\begin{abstract}
Summary
Age-related macular degeneration (AMD) usually affects both eyes over time. Among patients with advanced AMD in one eye estimates of the risk to the second eye have been diversely reported. Therefore we examined, over a four year period, the rate of second eye involvement in $\mathbf{4 1}$ patients with either exudative or advanced atrophic AMD in one eye, and early macular changes in the second eye with best corrected vision of $20 / 30$ or better. The cumulative risk of developing either exudative AMD or atrophic AMD and 20/80 or less vision in the second eye was $23 \%$ at four years. The cumulative risk of losing 10 or more letters on the ETDRS visual acuity chart in the second eye was $35 \%$ at four years. These results are discussed in relation to previously reported rates of second eye involvement in AMD.
\end{abstract}

Age-Related Macular Degeneration (AMD) is the leading cause of severe visual loss in persons over 60 years of age. ${ }^{1}$ It accounts for $13 \%$ of all cases of legal blindness in the United States. ${ }^{2}$ Both clinical forms of AMD, the exudative and the dry or geographic atrophy types have been well described. ${ }^{3-7}$ However, relatively little is known of their natural history. Macular drusen, sometimes in association with disturbance of the retinal pigment epithelium (RPE), are regarded as the earliest ophthalmoscopic manifestations of AMD. Furthermore, several clinical studies have established a definite association between macular drusen and visual loss due to AMD. ${ }^{4-5,8-11}$

Clinical studies have shown that AMD usually affects both eyes over time..$^{4,8-11}$ Thus, in patients with a unilateral advanced form of the disease it is of interest to be able to estimate the risk to the second eye. This risk had been diversely reported. Gregor et al. ${ }^{9}$ retrospectively studied a group of 104 patients, aged 60 to 69 years, who had unilateral disci- form AMD with subretinal neovascularisation or fluorescein angiography. They reported a $12-15 \%$ annual risk of developing a disciform lesion in the other eye. In another retrospective study, Bressler et $a l^{10}$ studied 38 patients with choroidal neovascularisation within the foveal avascular zone in one eye and macular drusen in the fellow eye. They found a $29 \%$ cumulative risk of developing a subretinal neovascular membrane in the second eye at three years. ${ }^{10}$ In a third retrospective study, Strahlman et al. ${ }^{11}$ studied 84 patients, aged 47 to 91 years, with unilateral exudative AMD and drusen only in the other macula. They reported a three to $7 \%$ risk per year of developing exudative AMD in the second eye for the three years following initial presentation. Differences in the severity of the macular changes and in the length of follow-up may, in part, explain discrepancies between studies.

At the Clinical Branch of the National Eye Institute (NEI) we had carried out a doublemasked randomised clinical trial of vitamins $\mathrm{C}$

From the Clinical Branch, National Eye Institute, National Institutes of Health, Bethesda, Maryland.

Correspondence to: Dr. M. Roy, University of Medicine and Dentistry, New Jersey Medical School, Department of Ophthalmology, 15 South Ninth Street, Newark NJ 07107, USA. 
and $\mathrm{E}$, or placebo, in AMD patients with an advanced form of the disease in one eye and early AMD changes with 20/30 or better best corrected visual acuity in the fellow eye. As part of the study the patients returned regularly to the eye clinic for follow-up examinations performed by masked observers. The study was initially planned for five years. Four years after the beginning of the study, event rates in the fellow eyes were examined for the two treatment groups. It then became evident that the sample size would have to be significantly increased thus requiring far more than one clinic to be involved. As a result, this phase of the study was terminated and the randomisation code broken. As we were interested in the risk of second eye involvement in patients with unilateral AMD, we decided to examine the rate of involvement of the fellow eye in patients revealed to have received placebo.

\section{Subjects and Methods}

Included in the NEI trial were 87 patients, aged 50-85 years, who had in one eye best corrected visual acuity reduced to $20 / 80$ or less due to either an atrophic or exudative AMD. Patients were either referred to the study by private ophthalmologists or self-referred following publicity for the trial. Those with exudative AMD had, on retinal fluorescein angiography, either a subretinal neovascular membrane or a serous detachment of the RPE in the macula greater than or equal to one disc diameter (DD) ${ }^{12}$ of confluent macular drusen within $1 / 2$ DD from the fovea, ${ }^{12}$ or a disciform scar of the macula (either spontaneous or secondary to laser photocoagulation). The other eye, the fellow or 'study eye', had either macular drusen, with or without localised hypo- or hyperpigmentation or atrophy of the RPE, but no evidence of subretinal neovascularisation or previous laser photocoagulation. In the study eye best distance visual acuity had to be $20 / 30$ or better. Excluded were aphakic patients, those with miotic pupils or lens changes precluding good visualisation of the retina, those with retinal vascular disorders, retinal degeneration, ocular hypertension or glaucoma. Also excluded were patients with poor general health and those over 85 years. It is of note that because of these restrictive ocular and systemic inclusion criteria far more patients than the 87 finally included in the study were actually examined.

Baseline evaluation included a detailed ocular and medical history. We recorded the age at diagnosis of the first eye involvement. Best corrected visual acuity in each eye for distance using ETDRS visual acuity charts and procedures, ${ }^{13}$ near vision and Amsler grid testing, were performed. A biomicroscopic examination of the anterior segment included recording the iris colour and the presence of either cortical or nuclear lens opacities. ${ }^{14}$ Iris photographs were obtained. Colour of the iris was noted as either "light" for blue, green and blue grey, or "dark" for hazel and brown irises. Intraocular pressure was measured by applanation. Retinal examination by direct and indirect ophthalmoscopy, and biomicroscopy was performed. Stereoscopic retinal colour photographs of fields one and two ${ }^{15}$ of both eyes were obtained using a Zeiss fundus camera. A retinal fluorescein angiogram centered on the macula in each eye was done, if a recent one was not available, to confirm eligibility of the patient.

Blood pressure was taken in the sitting position using a standard protocol. Patients who were receiving anti-hypertensive medications, and those who had either a systolic blood pressure $160 \mathrm{mmHg}$ or greater or diastolic pressure of $95 \mathrm{mmHg}$ or greater, ${ }^{16}$ were considered to be hypertensive. The presence of cardiovascular disease was ascertained from past medical history, absence of one or more peripheral pulses on clinical examination, and presence of electrocardiographic changes. We used the International Classification of Diseases, Eighth Revision (ICD 8 codes $410-414,430-437)$. Social class was determined using the classification of occupations of Goldthorpe and Hope. ${ }^{17}$

Eligible patients were randomly assigned to receive either vitamin $\mathrm{C}$ and $\mathrm{E}$ or placebo. All patients were given protective yellow glasses with a "cutoff" of $500 \mathrm{~nm}$ to wear all the time outdoors and indoors near fluorescent lights. All patients signed informed consent. Patients returned every four months for follow-up examinations, and were also seen whenever they experienced new symptoms. 
All visual acuity determinations and retinal examinations were done by masked observers.

Four-month follow-up visits included distance and near visual acuity with current glasses, Amsler grid testing, and retinal examination. If there was any change in the study eye from the previous examination, in either visual acuity or appearance of the Amsler grid, a subjective refraction was performed. Otherwise, best corrected visual acuity of the study eye and fundus photographs of retinal fields one and two were obtained on a yearly basis. In the worse eye the pathologic features of the macular area were classified into exudative, with or without subretinal neovascular membrane, or atrophic AMD. In the study eye we used the Wisconsin Age-Related Maculopathy grading system and scale of ascending severity (personal communication, Klein R, Davis M, Hubbard L, July 1989) for the colour photographs of retinal fields one and two. Patients were classified into:

(1). macular drusen (either small punctate or soft) only;

(2). RPE degeneration with soft drusen;

(3). geographic atrophy or RPE detachment.

In the statistical analysis, we examined the rates of events in the study eye using the Kaplan-Meier estimation technique. ${ }^{18}$ Here we report the data only for the 41 patients who at the end of the study, when the randomisation code was broken, were shown to have been on placebo throughout the study. We first considered the rates of "event" defined as the development in the study eye of either an exudative AMD, with or without subretinal neovascularisation, or an atrophic AMD reducing the distance best corrected visual acuity to $20 / 80$ or less. We also examined the rates of loss of 10 or more letters on the ETDRS visual acuity chart in the study eye. The minumum loss of 10 letters on the ETDRS chart was chosen arbitrarily in order to maximise the identification of patients with actual visual loss, while minimising the number of these with visual loss due to poor responses only. Student's t test was used to examine differences in demographic and ophthalmological characteristics between those with and those without event in the second eye.

\section{Results}

The demographic characteristics of the 41 AMD patients are shown in Table I. There were 21 men and 20 women. All patients were white except for one oriental. In their worse eye, 23 patients had an exudative AMD, of whom 13 had a subretinal neovascular membrane on fluorescein angiography, and a further ten had a disciform macular scar. Seven additional patients had macular scarring resulting from laser photocoagulation. Another two patients had a central RPE detachment greater than one DD without obvious subretinal neovessels on fluoresrein angiography. Nine other patients had an atrophic AMD in the worse eye.

In the study eye, 25 patients had macular drusen only. In 11 of the 25 patients the macular drusen were punctate and in 14 , they were soft. In addition to soft drusen, another 11 patients had some degree of RPE degeneration. Another five patients had areas of geographic atrophy sparing the fovea. Finally, two patients had soft drusen with an eccentric area 700 microns in its largest diameter of what could be classified as either confluent drusen or RPE detachment.

Over the four-year follow-up period, four of the 41 patients $(10 \%)$ died, and two $(5 \%)$ patients were lost after the first follow-up visit. Only one patient developed significant lens opacities in the study eye which necessi-

Table 1 Clinical characteristics of AMD patients

\begin{tabular}{ll}
\hline & $N=41$ \\
\hline Age (years) & $71.1(7.3)$ \\
Age at diagnosis first eye (years) & $68.0(8.0)$ \\
AMD in first or second degree relative & \\
$(\%)^{*}$ & 24 \\
Light iris (\%)* & 51 \\
Refractive error (diopters) & \\
Middle class & $0.56(2.5)$ \\
Lower class & 26 \\
Systolic Blood pressure (mm Hg) & 15 \\
Diastolic Blood pressure (mm Hg) & $78.5(20.5)$ \\
Systemic hypertension $(\%)$ & $37.4(8.2)$ \\
Cardiovascular disease (\%) & 68 \\
\hline
\end{tabular}

Means (SD)

* either visual loss due to AMD or macular drusen, diagnosed by an ophthalmologist.

* either blue, blue-grey or green, ${ }^{* *}$ spherical equivalent of the study eye.

+ ICD-8, International Classification of Diseases, Eighth Revision, codes 410-414, 430-437. 
tated cataract extraction, and resulted in 20/20 best corrected vision. After exclusion of the two patients lost to follow-up, all but four patients $(90 \%)$ wore the yellow glasses as recommended in the study protocol.

Of the seven patients who developed an exudative AMD in the study eye, five had a subretinal neovascular membrane, and two subretinal fluid and diffuse RPE staining of the macular area but no obvious subretinal neovascular membrane on retinal fluorescein angiography. One patient developed in the study eye geographic atrophy involving the centre of the macula. In these eight patients who developed an event in the study eye, the mean age at diagnosis of the second eye involvement was 71.1 (5.1) years, range 62-78 years. The average interval of time between events in the first and second eye was 3.6 (1.3) years, range 2 to 5 years.

The cumulative risk of developing either event was $10 \%$ at one and two years, $16 \%$ at three years, and $23 \%$ at four years (Fig. 1). There was no significant difference between those with and those without event in the second eye for either age at entry into the trial, or age at diagnosis of the first eye involvement, or refractive error of the study eye, or systemic blood pressure.

Over the four-year follow-up period, 12 patients lost ten or more letters on the ETDRS visual acuity chart. Visual loss was due to exudative AMD in seven patients, RPE degeneration or atrophy in four patients and confluent macular drusen in another patient. This represented a cumulative risk of $5 \%$ at one year, $13 \%$ at two years, $16 \%$ at three years, $35 \%$ at four years (Fig. 2).

\section{Discussion}

In the present study patients with unilateral advanced AMD had a cumulative risk to the second eye of either exudative or severe atrophic AMD of $16 \%$ at three years (and $23 \%$ at

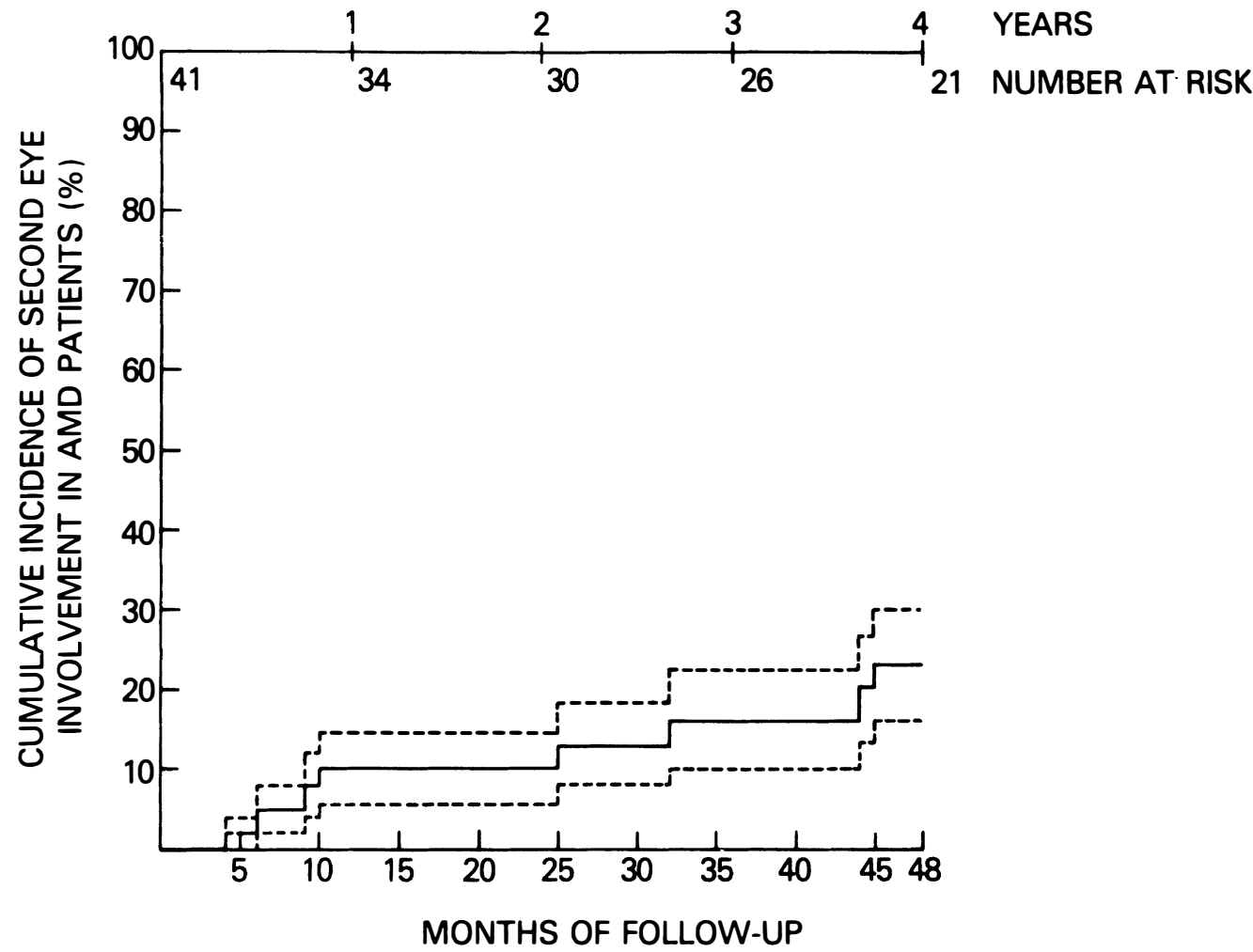

Fig. 1 Observed cumulative incidence (in percent) of any event, either 'exudative' or 'atrophic' $A M D$ in the second eye of patients who initially had unilateral advanced AMD. Dotted lines represent cumulative incidence \pm $S E$. The number of patients still at risk at each year is shown at the top of the figure. 


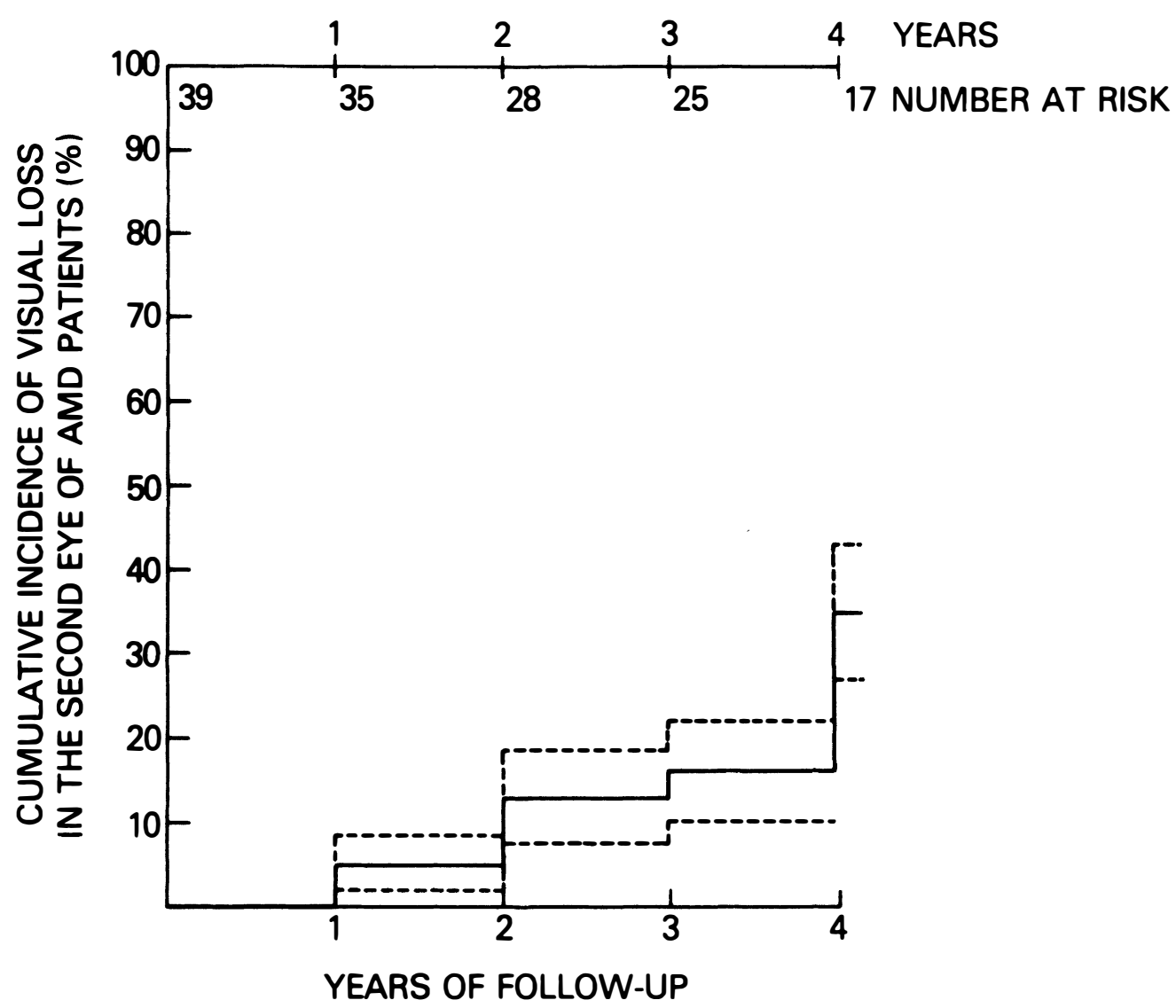

Fig. 2 Observed cumulative incidence (in percent) of visual loss of 10 letters or more ETDRS visual acuity chart in the second eye of patients who initially had unilateral advanced AMD. Dotted lines represent cumulative incidence $\pm S E$. The number of patients still at risk at each year is shown at the top of the figure.

four years). The three year prospective cumulative risk of $16 \%$ was similar to the $17 \%$ reported in the retrospective study of Strahlman et al. ${ }^{11}$ but somewhat less than the $32 \%$ reported by Gregor et al. ${ }^{9}$ or the $29 \%$ found by Bressler et al. ${ }^{10}$ in their retrospective studies. In the present study the average interval of time between events in first and second eye (3.6 years) was similar to the 3.3 years reported by Gass for the 31 patients who developed a disciform lesion in their second eye. ${ }^{4}$

With regards to visual loss of ten or more letters in the second eye, the $13 \%$ cumulative risk at two years in the present study is also lower than the $34 \%$ two year incidence rate reported for the control group in Newsome et al's study. ${ }^{19}$ However, in that study control patients may have had in the study eye more advanced AMD changes than study eyes included in the present study. For instance, at entry $68 \%$ of their control patients had a visual acuity worse than $20 / 25,{ }^{19}$ while all our patients had $20 / 30$ or better. Interestingly, in that randomised clinical trial the frequency of visual loss of ten letters or more (using an averaging method) in the zinc-treated AMD patients was $14 \%$ at two years, ${ }^{19}$ which is similar to that found in the present study.

It is possible that our lower rates of either event or visual loss may be related to sampling variation. However, our group of patients appears comparable to those previously reported $^{9,11}$ with regards to age, family history of AMD and cardiovascular complications. Another possibility is that the wearing of the yellow glasses, which were worn very consistently by $90 \%$ of the patients may have had 
some protective effect in our patients. There is clinical and experimental evidence (reviewed $\mathrm{in}^{20}$ ) to suggest a possible toxic effect of light, particularly UV and blue light ${ }^{21}$ on the retina. It has been hypothesised that photons of light initiate the liberation of oxygen-derived free radicals in the retina,${ }^{22}$ which in turn lead to chain auto-oxidation. Thus, the wearing of glasses filtering out all wavelengths below $500 \mathrm{~nm}$ may reduce the amount of free radicals generated in this fashion.

In summary, the results of the present study indicate that the rate of involvement or of visual loss in the second eye of patients with an advanced form of AMD in the other eye may be substantially less than that previously reported. However, ours was a referred sample, and these data need to be confirmed in prospective studies with a larger number of AMD patients. Nonetheless, these preliminary data suggesting a lower event rate may be of some relief to the growing number of AMD patients afflicted with unilateral severe visual loss. This lower risk, if replicated, may also impact on sample size and length of follow-up needed to assess efficacy of treatments in randomised clinical trials of AMD.

We gratefully acknowledge Carl Kupfer, M.D. for initiating the National Eye Institute Age-Related Macular Degeneration Study and providing helpful advice with the manuscript. Thanks are also due to Sanford Meyers, M.D. who was involved in the designing and writing of the original protocol, to Ernest Kuehl and Pat Ciatto for the fundus photographs, to Marvin Podgor M.S. and Valerie Friedlin Ph.D. for statistical help, and to Jane Armstrong from the Fundus Photograph Reading Center at the University of Wisconsin-Madison for the grading of the fundus photographs.

\footnotetext{
References

${ }^{1}$ Kahn H and Moorhead H: Statistics on blindness in the Model Reporting Area, 1969-1970 Washington, D. C., DHEW, PHS Publication (NIH) 73427, USGPO 1973.

${ }^{2}$ Ferris FL III, Fine SL, Hyman LG: Age-related macular degeneration and blindness due to neovascular maculopathy. Arch Ophthalmol 1984; 102: $1640-2$.

${ }^{3}$ Junius $\mathrm{P}$ and Kuhnt $\mathrm{H}$ : Die Scheibinformige Entartung der Netzhautmitte. Berlin: S Karger, 1926; 77.

${ }^{4}$ Gass JDM: Drusen and disciform macular detachment and degeneration. Arch Ophthalmol 1973; 90: $206-17$.
}

${ }^{5}$ Teeters VW and Bird AC: The development of neovascularization of senile disciform macular degeneration. Am J Ophthalmol 1973; 76: 1-18.

${ }^{6}$ Blair C: Geographic atrophy of the retinal pigment epithelium. A manifestation of senile macular degeneration. Arch Ophthalmol 1975; 93: 19-25.

${ }^{7}$ Willerson D Jr and Aaberg TM: Senile macular degeneration and geographic atrophy of the retinal pigment epithelium. $\mathrm{Br}$ J Opthalmol 1978; 62:1 551-3.

${ }^{8}$ Chandra SR, Gragoudas ES, Friedman E, Van Buskirk EM, Klein ML: Natural history of disciform degeneration of the macula. Am J Ophthalmol 1974; 78; 579-82.

${ }^{9}$ Gregor Z, Bird AC, Chisholm IH. Senile disciform macular degeneration in the second eye. $\mathrm{Br} J$ Ophthalmol 1977; 61: 141-7.

${ }^{10}$ Bressler SB, Bressler NM, Fine SL, Hillis A, Murphy R, Olk RJ, Patz A: Natural course of choroidal neovascular membranes within the foveal avascular zone in senile macular degeneration. Am J Ophthalmol 1982; 93: 157-63.

${ }^{11}$ Strahlman ER, Fine SL, Hills A: The second eye of patients with senile macular degeneration. Arch Ophthalmol 1983; 101: 1191-3.

${ }^{12}$ Meredith TA, Braley RE, Aaberg TM: Natural history of serous detachment of the retinal pigment epithelium. Am J Ophthalmol 1979; 88: 643-51.

${ }^{13}$ Ferris FL III, Kassoff A, Bresnick GH, et al: New visual acuity charts for clinical research. Am J Ophthalmol 1982; 94: 91-6.

${ }^{14}$ Chylack LT, Leske C, Sperduto R, Khu P, McCarthy $\mathrm{D}$ and the Lens Opacities Case-Control Study Group. Lens opacities classification system. Arch Ophthalmol 1988; 106: 330-4.

${ }^{15}$ Diabetic Retinopathy Study Research Group: Diabetic Retinopathy Study Report Number 7: A modification of the Airlie House classification of diabetic retinopathy. Invest Ophthalmol Vis Sci 1981; 21 : 210-36.

${ }^{16}$ Goldthorpe JH and Hope K: The social grading of occupation: a new approach and scale. New York, Oxford University Press, 1974: 134-43.

${ }^{17}$ Schurleff D: Some characteristics related to the incidence of cardiovascular disease and death: Framingham Study, 12 year follow-up: Section 30, in Kannel WB, Gordon T (eds): the Framingham Study: An Epidemiological Investigation of Cardiovascular Disease, Public Health Service publication (NIH) 74-599. US Department of Health Education and Welfare, 1974; pp 1-32.

${ }^{18}$ Kaplan EL and Meier D: Nonparametric estimation from incomplete observations. J Am Statist Assoc 1958; 53: 457-81.

${ }^{19}$ Newsome DA, Schwartz M, Leone NC, Elston RC, Miller E: Oral zinc in macular degeneration. Arch Ophthalmol 1988; 106: 192-8.

${ }^{20}$ Young RW: Solar radiation and age-related macular degeneration. Surv Ophthalmol 1988; 32: 252-69.

${ }^{21}$ Ham W, Mueller H, Sliney D: Retinal sensitivity to damage from short wavelength light. Nature 1985; 260: 615-27.

${ }^{22}$ Feeney L and Berman ER: Oxygen toxicity membrane damage by free radicals. Invest $O$ phthalmol 1976; 15: 789-92. 